\title{
Analyzing Barriers to Digital Transformation in the German Engineering Industry - A Comparison of Digitalized and Non-Digitalized Enterprises
}

\author{
Henning Brink \\ Osnabrück University \\ henning.brink@uni-osnabrueck.de
}

\author{
Sven Packmohr \\ Malmö University \\ sven.packmohr@mau.se
}

\begin{abstract}
The German engineering sector is one of the most prominent industries in Germany in terms of revenues, the number of employees, and reputation for the "Made in Germany" brand. In this industry, digital transformation (DT) has become a significant trend. DT is more than optimizing internal processes by digital means. It entails the offer of digital services and products and the enhancement of customer experience. Complex barriers need to be overcome to drive this transformation forward. Therefore, our study analysis the organizational barriers to DT within the German engineering industry. We follow a quantitative approach to gain insight on organizational barriers by a comparison of digitalized and less digitalized enterprises and their DTs. Our research demonstrates that digitalized enterprises perceive lower degrees of certain barriers in leadership, culture, employees, and skills, which are essential parts in a socio-technical view. However, there are still barriers that digitalized enterprises are struggling with.
\end{abstract}

\section{Introduction}

Digital transformation (DT) has an ubiquitous impact [1]. It will fundamentally change the way enterprises and societies operate and collaborate. Digital goods and services have become the norm [2]. Enterprises that are not adhering to this norm, lose a source of competitive advantage. Research describes DT as the reinforcement of an enterprise's competitive advantage [3] through workplace improvements [4], or successful implementation of business models [5]. Nevertheless, organizations struggle with the opportunities and threats introduced by DT [6]. DT is a complex phenomenon. The comprehension of the wide variety of technologies, stakeholders, and differences between the application areas is challenging. Effects differ based on the intensity of interaction between the individuals and technology and interfere with the progress [7]. In the present study, we define all negative effects, difficulties, challenges, issues, or problems as barriers. Barriers are "those things that hinder, slow, or stop the process of DT" [8].

In particular, the impact of organizational barriers is significant on the DT within enterprises. Vial [7] drafted conceptual building blocks of a DT. He linked organizational barriers and structural changes to the changes in the value creation paths. Thus, our work focuses on organizational barriers that affect the DT of companies. These barriers are especially critical since the organizational structure aims to solve the tension between technological opportunities and strategic requirements [9]. Decision-makers should be informed about potential threats to DT. Knowing their origins and describing them [10] would help managers intervene and apply adequate solutions. Thus, our research question is determined as follows: What is the difference in organizational barriers between two subsets of German engineering enterprises with differing degrees of digitalization?

This research contributes to the development and testing of barrier models. As DT is an evolving field, qualitative methods are prominent. Our approach follows a call for more quantitative research within digitally related subjects [11] to validate the impact of barriers. A more rigorous approach can help decisionmakers in companies to identify critical barriers and implement solutions.

The article aims to measure the impact of various organizational barriers on DT of enterprises being active in the German manufacturing sector. The manufacturing sector is a significant source of wealth for the German economy. During 2020, it generated $€ 2.1$ billion in revenues, employed 7.4 million individuals, and the staff expenditure rate was $18.9 \%$ in the industry [12]. According to the German Industry 4.0 initiative [13], companies in this sector are pushed in their DTs.

In a pre-study, we investigated the barriers affecting a DT [8]. The study included 46 qualitative interviews with users and decision-makers working in German 
engineering enterprises and being experienced in the field of DT. Based on the collected data, we converted barriers found into hypotheses and measurement scale items with an organizational approach [14]. Since the tension between technological and social factors lead to organizational barriers, we adopted a socio-technical approach $[15,16]$. The quantitative study data were collected with the above-mentioned scale from 151 participants working in German engineering enterprises. Then, the data was divided into subsets of digitalized and non (fully) digitalized enterprises to analyze their differences. The focus on one sector allows us to isolate specific experiences of companies within German engineering. The statistical analyses were conducted on the findings with the Mann-Whitney $U$ test to determine the differences between the subsets.

In the next section, we will present the theoretical framework of the study. We will introduce the research approach, followed by the data collection results. Then, these results will be discussed, and the conclusion section will include the contribution and limitations of our findings.

\section{Theoretical development}

DT has become a buzzword with a multitude of definitions. Reis et al. [17] aggregated various definitions of DT into "the use of new digital technologies that enable major business improvements and influences all aspects of customers' lives." Technology, business, and customers are the three farreaching elements, indicating the complexity of DT. Vial [7] decomposed several definitions of DT and identified the four elements as target, scope, means, and outcome. Based on these elements, he defined DT as "a process that aims to improve an entity by triggering significant changes to its properties through combinations of information, computing, communication, and connectivity technologies." To further explain the concept of DT, Vial [7] defined eight building blocks. The most central element was determined as the changes in value creation. These changes are enabled by the employment of digital technologies but are also affected by structural changes and organizational barriers. A related term to DT is ITEnabled Organizational Transformation (ITOT). The difference between both is that DT is inducing a new organizational identity because digital technologies reshape an organization's business model. In an ITOT digital technology is supporting existing business models leading to a reinforced organizational identity [18]. Thus, DT is the more complex endeavor and requires a more holistic approach. As it "changes business elements, including strategy, business model, business processes, organizational structures and organizational culture" [19]. A multidimensional framework of DT links contextual conditions, mechanisms, and outcomes. Contextual conditions are to a high degree external and trigger mechanisms within an organization leading to outcomes for the organization and side-effects for other stakeholders [20]. Thus, within the mechanisms, there might be frictions.

To measure the degree of a DT, researchers developed maturity models. Within manufacturing, digital technologies can be used to enhance production techniques or they can be built into the product. If the production shifts focus to a more digitally-supported one and if the orientation within the value creation becomes more service-oriented, a fully digital transformed enterprise evolves. The maturity of the product realization and product application can range from being digitally aware until being a data-driven enterprise [21]. This observation coincides with the distinction between ITOT and DT. Awareness as an ITOT-approach might lead to discrete enhancements of business models and reinforce an organization. Whereas DT provokes a profound change in business models and a renewal of organizational identity [18].

DT presents both a threat and an opportunity for existing organizations and should be managed adequately [10]. Leaders should consider the obstacles and facilitators to guide the organization through the DT process. Close observation of the barriers is especially critical, as they might obstruct or slow down the DT process [8]. Initial study findings on barriers were conducted in the field of innovation research [22], which evolved into DT research. Often, barriers are associated with a specific technology and are represented as simple lists. E.g., Bilgeri and Wortmann [23] identified a list of barriers that obstructed the employment of Internet of Things technology. In some cases, researchers developed certain barrier classifications in different contexts by aggregating data into clusters [24, 25]. Comparing six different studies on barriers to DT, the organizational aspect plays a major role. Interestingly, the technical aspect is not as present [26]. Often, internal and external views revealed a pattern within barrier classifications. Internal classifications represent obstacles associated with resources and supply, culture, and people, time, and IT systems. Also, management issues, such as strategy and organization, were represented [27]. Barriers are used as a concept in technology-enhanced business models [28, 29].

Managers should scrutinize the barriers to DT and find ways to overcome them. The key is to build up capabilities [30] that address the barriers and turn them into facilitators. Corporations can acquire a competitive advantage by the employment of these facilitators. Often, one approach is to focus on positive factors such as strengths and opportunities in strategic planning to 
gain a competitive advantage [31]. As DT is ubiquitous with complex barriers and facilitators, an ambidextrous approach should be considered. Barriers and facilitators are interrelated. If the intra-organizational DT process evolves or external conditions change, barriers may turn into facilitators [32]. Research on both barriers and facilitators is of equal importance to achieve a holistic perspective towards the evolution of DT processes.

The barriers concept has been applied in various fields of research. Frequently, results are shaped by the employment of a specific technological lens or specific stakeholders [33]. Based on a list of barriers and initial solutions, researchers investigated the methods that different organizations considered in the adoption of new technologies. In our study, we derive hypotheses from a list of organizational barriers, convert these into items, collect the associated data, and test the data against the hypotheses. We applied this research procedure to data from the engineering industry. By focusing on a specific industry, we could compare the findings of our previous and our present study.

\section{Research design}

In the present study, we built on existing qualitative results from a pre-study. With the help of the qualitative results, we developed hypotheses and constructed a questionnaire. In a quantitative phase, we then tested our hypotheses using this questionnaire.

\subsection{Pre-study}

We conducted 46 semi-structured interviews with corporate DT experts using an interview manual developed in a previous study [8]. The narrativeoriented questions aimed to determine the DT status general and the barriers to DT based on the interviewee's perspective. The sample was assigned with the purposive sampling method [34] based on personal networks and professional network sites. Respondents who were employed in various positions were assigned. The 46 interviewees were employed in 31 enterprises, mostly in the manufacturing sector and automotive, agricultural engineering, plastics, and steel sector. Also, manufacturing services and consulting services firms were included in the sample. An overview of the interview sample is presented in Table 1.

We transcribed and openly coded the data from the interviews with the web-based tool Qcamap [35]. In the process, one hundred and eighty open codes about barriers were identified. The codes were iteratively revised and clustered. As a result, external, organizational, individual, and technical barriers were derived based on the interview clusters.
To improve the interview findings, we focused on the quantitative analysis of the organizational barriers in greater detail. We derived hypotheses and questionnaire items based on the organizational barrier cluster elements [36]. For the item development, we oriented towards the proposed procedure by MacKenzie et al. [14] and double-checked existing scales for organizational change [37].

The developed questionnaire included a section where the socio-demographic attributes of the participants were asked. The main section aimed at determining the participant perceptions on the DT and the organizational barriers. It included 14 items on the DT process and DT barriers measured on a 5-point Likert-type ranging from "I disagree" to "I agree".

To gain deeper insights, participants had the opportunity to give open-ended answers in the questionnaire on their perceived barriers more specifically. We will pick up on these in the later discussion for a better interpretation of the quantitative results.

\section{Table 1. Interview sample}

\begin{tabular}{|l|l|l|}
\hline Industry & Positions & N \\
\hline $\begin{array}{l}\text { Auto- } \\
\text { motive }\end{array}$ & $\begin{array}{l}\text { Head of R\&D, Engineer, Digital } \\
\text { Manager, Managing Director }\end{array}$ & 14 \\
\hline $\begin{array}{l}\text { Agri- } \\
\text { culture }\end{array}$ & $\begin{array}{l}\text { Head of Quality Management, } \\
\text { Managing Director, IT Manager, } \\
\text { Operations Manager, }\end{array}$ & 9 \\
\hline $\begin{array}{l}\text { Plastics } \\
\text { Industry }\end{array}$ & $\begin{array}{l}\text { Head of Production, Head of } \\
\text { R\&D, Shift Supervisor, } \\
\text { Engineer }\end{array}$ & 5 \\
\hline $\begin{array}{l}\text { Steel } \\
\text { Industry }\end{array}$ & $\begin{array}{l}\text { Managing Director, Head of } \\
\text { Production Intelligence, Head of } \\
\text { Product and Innovation }\end{array}$ & 4 \\
\hline Services & $\begin{array}{l}\text { Information Manager, IT } \\
\text { Support, Managing Director }\end{array}$ & 3 \\
\hline Consulting & Consultant & 3 \\
\hline $\begin{array}{l}\text { Other } \\
\text { Manu- } \\
\text { facturing }\end{array}$ & $\begin{array}{l}\text { Business Development } \\
\text { Manager, (Deputy) Operations } \\
\text { Manager, Chief Technical } \\
\text { Officer, Head of Production }\end{array}$ & \\
\hline
\end{tabular}

\subsection{Main study}

To collect the quantitative data, we called professional social media network members and asked for their participation. The shout-outs were specifically directed at employees in the automotive industry as well as in the mechanical and plant engineering sectors. These sectors traditionally represent the core of the 
German economy [12]. Also, they are the focus of the German Industry 4.0 initiative [13]. Thus, these sectors benefit from major initiatives to improve digitalization. In the period between December 2019 and May 2020, 151 individuals employed in the listed industries responded to our call and completed the questionnaire. The voluntary, anonymous, and standardized questionnaire could be answered online.

To obtain a comprehensive insight into the manufacturing industry, the questionnaire addressed all levels of the employee hierarchy. The sample included employees who worked as executive managers, employees with management responsibility, employees without management responsibility, or interns. The sample included a mix of employees from small, midsize, and large corporations. This diversified sample allowed us to learn as much as possible [38]. A detailed overview of the sample can be found in Table 2 .

After the data were collected, we prepared it for the later statistical analysis. In the process, we re-coded the Likert scale into an interval scale. The distances between the Likert scale grades were accepted as equal to improve the statistical analysis. We also poled the answers to the items in a uniform direction. This was necessary since the questionnaire items included both positive and negative statements to avoid bias.

To check the reliability of the questionnaire we calculated Cronbach's alpha. Determining the internal consistency of the dimensions provided information on whether we could aggregate the items within the respective dimensions and in turn apply the dimensions to further statistical analysis [39].

After that we divided the sample into two different subsets, to achieve a deeper understanding of the barriers of DT and to answer the research questions. As described in the study of the manufacturing sector by Klötzer and Pflaum [21], DT can be assessed by an enterprise's smart product realization and application. Based on a self-assessment by the participants, we divided the sample into digitalized and partial digitalized enterprises. The subset of digitalized enterprises ( 55 participants) is defined by both realizing and applying smart products. The subset of enterprises with no or partial digitalization (96 participants) lacks at least one of the constituting elements. Hereafter, the latter subset is abbreviated simply as "non-digitalized enterprises". As classifier for a digitalized enterprise, we relied on two items within the DT dimension (see Table $3)$.

Since we assumed that the DT slowed down or was prevented by the barriers identified in qualitative research, participants in the subsets should perceive these barriers in different intensities. Nevertheless, it is unclear whether DT is equally slowed by all barriers, or whether there are particularly salient barriers. To examine the differences, we conducted a Mann-Whitney $\mathrm{U}$ test as the quantitative data showed a non-normal distribution according to the Shapiro-Wilk test. The Mann-Whitney U test was chosen because it compares the medians of the subsets, determines if there significant differences and allows a calculation of the specific effect sizes [40]. As other studies have shown, comparing digitalized with non-digitalized enterprises using the Mann-Whitney $U$ test, can contribute to a better understanding of the research subject [41].

\section{Results}

In the first part of the results section, we will describe the development of the hypotheses stemming from qualitative data. In the second part, we will revise the statistical results.

\subsection{The underlying barrier model and related questionnaire}

The description of DT in our underlying barrier model was based on the research by Klötzer and Pflaum [21]. The process of DT can be determined from two perspectives. The first perspective is the development of smart products, which focuses on the creation of new and innovative DT-induced products for the customers. The second perspective is associated with the adoption of smart products in the corporate value chain to optimize production. Fully digitalized enterprises consider both perspectives in their DT.

We measured the DT process based on two items associated with smart product realization and application [21]. In the operationalization, a distinction was made between the existence of a portfolio of smart products and the existence of largely digitalized business processes.

On the way to become digitally transformed, enterprises may encounter various organizational barriers that can slow down or even prevent changes. We openly coded the interview data and aggregated these codes into characteristics. Based on these characteristics, we formulated the items. We also matched the characteristics with Vial's [7] DT building blocks to provide a dimensional context. Overall, we determined four dimensions that impact the corporate DT process based on the above-mentioned analytic procedure. In the following section, the construction of the items and the connection between the dimensions and Vial's building blocks are discussed.

Based on our interview data, we coded the statements about leadership barriers. It is an important task for organizational leaders to develop the organization's digital attitudes [7]. 
Table 2. Questionnaire sample

\begin{tabular}{|l|l|l|l|l|l|}
\hline \multicolumn{2}{|l|}{ Distribution of industries } & Distribution of positions & \multicolumn{2}{l|}{ Distribution of the enterprise size } \\
\hline Automotive & $72 \%$ & Executive Manager & $5 \%$ & 1000 or more & $30 \%$ \\
\hline $\begin{array}{l}\text { Mechanical \& } \\
\text { plant engineering }\end{array}$ & $28 \%$ & $\begin{array}{l}\text { Employee with management } \\
\text { responsibility }\end{array}$ & $25 \%$ & $250-999$ & $7 \%$ \\
\hline \multirow{5}{*}{} & $\begin{array}{l}\text { Employee without management } \\
\text { responsibility }\end{array}$ & $57 \%$ & $50-249$ & $48 \%$ \\
\cline { 2 - 6 } & Intern & $9 \%$ & $10-49$ & $10 \%$ \\
\cline { 2 - 5 } & Other & $4 \%$ & 9 or less & $5 \%$ \\
\hline
\end{tabular}

Interviewee 29 (I29) mentioned a situation where "50\% of the people involved in the process did not understand that the topic [DT] was strategic. That was the limiting factor." Therefore, we surveyed how the communication of the DT strategy was perceived. Another aspect was the perception that "the top management needs to participate" (I21). Thus, we measured the degree of visible top management involvement. An aspect of leadership is to let employees participate in decision-making. In a previous study, the "Business Case for the digitalization of the production" was developed (I28). Building on these aspects of the interviews, we measured the likeliness of employee involvement in the decisions about the implementation of new technologies. Our items pointed towards the construction of capabilities to respond to possible contingencies in the employment of digital technologies. New roles such as Chief Digital Officer (CDO) or a specific department should be introduced to undertake the construction of these capabilities. Like IT, DT requires a strategic perspective and alignment with business objectives. Digital business strategies should be broken down into specific courses of action, which would impact corporate organization and processes. IT and business functions need to work together on transformation to allow these courses of action. Certain resources indicate that the role of CDO is temporary. A transformation is also temporary and will reach a stable situation [7]. Based on the leadership barriers we found, we assumed:

Hypotheses 1 (H1): Non-digitalized enterprises perceive significantly higher leadership barriers when compared to digitalized enterprises.

I10 draws comparisons to a start-up culture: "For a corporation also, it is very crucial making, accepting and forgiving mistakes." Thus, errors should be used to improve work processes. An agile approach might lead to constant learning and improvement. "You might need a Minimal Viable Product if you want to prove a hypothesis. If it doesn't work that way and you have to change it again" (I10). Another factor is the openness to new ideas about the digitalization of the processes, methods, techniques. "That's why there is a lot of movement in the company and a great deal of openness. We are preparing to modernize many places. That is why we look specifically at the new technologies' industry in Silicon Valley to find new business models and completely new approaches in corporate management" (I08). The disruption caused by DT affects the organizational culture. Often, firms exhibit an organizational separation between IT and business functions, which became an integral part of the organizational values and beliefs. Research demonstrated that the development of digital capabilities requires an innovative culture, a common language, and expertise in value creation technology. Organizations should learn how to experiment and take risks. On a small scale, experiments with digital technologies need to be conducted and, in the case of success, scale up to the rest of the organization. Such an agile procedure draws from software development experiences. Organizations conduct small, incremental, and iterative steps. Based on the results of these steps, long-term goals should be adapted. Furthermore, changes in the environment should be monitored [7]. Therefore, we assumed that:

Hypotheses 2 (H2): Non-digitalized enterprises perceive significantly higher cultural barriers when compared to digitalized enterprises.

If no new roles emerge, it would hardly be possible to address the changed requirements. I22 perceived a barrier within "Possessions, old understanding of roles. That certainly stands in the way of digitalization". Thus, we surveyed the item for a lack of new roles to cope with DT. Connected to the roles, there is a shortage of both financial and human resources. In addition to the roles, respondents experienced a general lack of resources. "If I want to digitalize my service, I need certain resources, skills that can also support the digitalized service, whatever it looks like" (I21). 
Table 3. Questionnaire

\begin{tabular}{|l|l|l|l|l|}
\hline Dimension & Item in keywords & $\begin{array}{l}\text { Mean } \\
\text { non-digitalized } \\
\text { enterprises }\end{array}$ & $\begin{array}{l}\text { Mean } \\
\text { digitalized } \\
\text { enterprises }\end{array}$ & Delta \\
\hline \multirow{2}{*}{ DT } & Portfolio of smart products or services for customers & 2.71 & 4.31 & 1.60 \\
\cline { 2 - 5 } & Digital support for business processes & 3.10 & 4.41 & 1.31 \\
\hline \multirow{5}{*}{ Leadership } & $\begin{array}{l}\text { Senior management is not supportive \& not visibly } \\
\text { engaged }\end{array}$ & 2.63 & 1.93 & -0.70 \\
\cline { 2 - 5 } & Clear DT strategy not communicated & 3.26 & 2.19 & -1.07 \\
\cline { 2 - 5 } & $\begin{array}{l}\text { Employees not involved in decisions on technology } \\
\text { implementation }\end{array}$ & 3.32 & 2.95 & -0.37 \\
\hline \multirow{5}{*}{ Eulture } & Errors not used to improve work processes & 2.61 & 2.35 & -0.26 \\
\cline { 2 - 5 } & No constant learning and improvement & 2.63 & 1.95 & -0.68 \\
\cline { 2 - 5 } & $\begin{array}{l}\text { No openness to new ideas about DT in processes, } \\
\text { Emplodods, techniques, etc. }\end{array}$ & 2.36 & 1.83 & -0.53 \\
\hline \multirow{3}{*}{ Skills } & No new roles to manage DT projects. & 2.88 & 2.00 & -0.88 \\
\cline { 2 - 5 } & Not enough resources and staff to manage DT & 3.05 & 2.70 & -0.35 \\
\cline { 2 - 5 } & IT knowledge is not adequate to keep up with DT & 2.90 & 2.28 & -0.62 \\
\cline { 2 - 5 } & Lack of knowledge about the potential of DT & 3.21 & 2.57 & -0.64 \\
\cline { 2 - 5 } & Lack of knowledge to use digital tech effectively & 3.77 & 3.71 & -0.06 \\
\cline { 2 - 5 } & Lack of digital tech training for the employees & 4.09 & 3.88 & -0.21 \\
\hline
\end{tabular}

Such statements lead to the question of how the enterprises were equipped with resources and staff to manage DT. Changes in the organizational structure and culture allow for new roles and responsibilities. As IT and business functions need to move closer, opportunities arise for business employees to lead digital developments. In contrast, IT employees cooperate in such developments [7].

Hypotheses 3 (H3): Non-digitalized enterprises perceive significantly higher employee barriers when compared to digitalized enterprises.

The last dimension, missing skills, entails the absence of specific knowledge, expertise, and competencies in the DT domain. "Of course, problems arise at this point. Different occupational levels simply have to adapt first. Assertion of the importance of IT technology directly on the employee, who then also provides the service, is not that easy." (I37) Thus, we investigated whether the employees felt that their IT knowledge was adequate to keep up with DT. I23 felt that "just the step now that everyone can do and use it company-wide and recognize the potential" was missing. Based on these codes, we derived that there was a lack of knowledge about the potential of DT. One of the important achievements was efficiency. "If you know, e.g., how can a company increase its efficiency, then that is a very nice approach [to DT]" (I21). The barrier to address statements in this group of codes was the lack of knowledge on how to use digital technologies effectively. Certain respondents recognized training problems. "We have a very high training expenditure at the beginning. We need equipment. We need to rethink. Of course, it's a timing problem, even with large enterprises like us at this point" (I37). Based on the codes associated with training, we generated an item on whether more training was required to improve employees' technological knowledge. New technologies require new skills from current and future employees. Especially, analytical and complex problem-solving skills are required. Human resources are responsible for the improvement of these skills and providing employee assistance to adopt DT challenges [7]. Thus, we hypothesized the following:

Hypotheses 4 (H4): Non-digitalized enterprises perceive significantly higher skill barriers when compared to digitalized enterprises.

The above-described dimensions were transferred into a questionnaire and presented in Table 3.

\subsection{Statistical analysis}

After the quantitative data collection, we aggregated the items in their respective dimensions and tested the interview-based dimensions for internal consistency. Thus, we conducted a reliability analysis with Cronbach's alpha coefficient. The values ranged between 0.60 and 0.73 (see Table 4). These values are reported in the literature as being moderate up to acceptable [39]. An exception was the employee 
dimension with a coefficient of 0.43 , indicating rather weak reliability.

Since the dimension included only two items and deletion of these items would not yield an increase in internal consistency, we continued with the analysis. However, examining also the results at the item level and not just focusing at the dimensional level can overcome the deficiency of the low Cronbach's alpha value to a certain extent in our study.

In the next step, we divided the sample into two subsets to test our hypotheses. We expected the subset with non-digitalized enterprises would exhibit a slower transformation due to organizational barriers, and participants should, in turn, observe higher degrees of barriers. Using the Mann-Whitney U test, we investigated whether there were statistically significant differences [42] in the perception of the barriers between the subsets.

Starting with the leadership dimension, there was a significant difference between the two subsets (see Table 4). Furthermore, a moderate effect size (0.42) was calculated. Among all barrier dimensions, the strongest effect size was observed here. The effect size indicates the extent to which the responses of the subsets overlap [43]. The higher the effect size, the lower the overlap. An effect of 0.10 is considered weak, an effect of 0.30 is considered moderate, and an effect of 0.50 is considered high based on the differences between the subsets [44]. Extreme differences were less visible at the aggregated dimension level. Therefore, we also analyzed the differences on the item level to check whether there was a clear pattern at the item level as well. The review of the specific items revealed that the biggest differences were in the lack of senior management support and the lack of clear communication of DT strategy dimensions. Based on the Mann-Whitney $U$ test results, we accepted hypothesis 1 that non-digitalized enterprises perceive significantly higher leadership barriers when compared to digitalized enterprises.
A moderate effect size of 0.31 and a significant difference based on the Mann-Whitney U test could also be observed in culture. Across the subsets, this dimension exhibited the lowest mean values, which indicated that the cultural dimension was perceived to have a lower degree of intensity when compared to leadership, employee, and skills dimensions. Higher differences can only be observed in constant learning and improvement in this dimension. Nevertheless, we can accept hypothesis 2 .

For the third barrier dimension, employees, a significant difference, and moderate effect size were also evident. At the item level, the largest difference in the response behavior of the two subsets was particularly apparent in the new roles in DT project management. Based on the Mann-Whitney U test, hypothesis 3 could therefore be accepted.

The skills dimension also demonstrated significant differences between the subsets. However, it shows the lowest effect size across all barrier dimensions. It was noticeable that the skills dimension exhibited the highest mean values in both subsets. Regardless, the lack of training was perceived as a major problem. The lack of knowledge on DT's potential, on the other hand, demonstrated the highest difference (0.64), indicating that non-digitalized enterprises were more affected.

Overall, all Mann-Whitney U test results showed significant differences and weak to moderate effect sizes, which proved that the two subsets were different [45]. A clear pattern could be observed in the barrier dimensions and on item level. Participants of digitalized enterprises perceived lower barriers in general. However, the differences were not equally significant for each barrier. Based on the findings, we accepted our hypotheses H1, H2, H3, and H4: Non-digitalized enterprises perceive significantly higher leadership, culture, employee, and skill barriers when compared to digitalized enterprises (and vice versa).

\section{Table 4. Statistical analysis}

\begin{tabular}{|c|c|c|c|c|c|c|c|c|c|c|c|c|c|c|c|}
\hline \multirow[t]{2}{*}{ Dimension } & \multirow[t]{2}{*}{$\begin{array}{l}\text { Cronbach's } \\
\text { Alpha }\end{array}$} & \multicolumn{2}{|c|}{$\begin{array}{l}\text { Mann-Whitney } \\
\text { U test }\end{array}$} & \multicolumn{6}{|c|}{ Non-digitalized enterprises subset } & \multicolumn{6}{|c|}{ Digitalized enterprises subset } \\
\hline & & 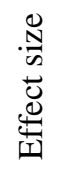 & $\dot{00}$ & Z & 志 & 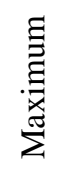 & $\sum^{\Xi}$ & 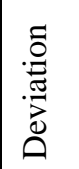 & 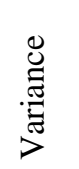 & Z & $\underset{\Xi}{\Xi}$ & 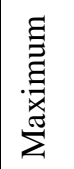 & $\sum^{\Xi}$ & 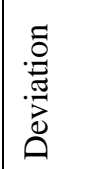 & 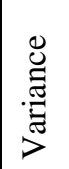 \\
\hline Leadership & 0.60 & 0.42 & 0.00 & 96 & 1.33 & 5.00 & 3.07 & 0.82 & 0.67 & 55 & 1.00 & 3.67 & 2.36 & 0.64 & 0.41 \\
\hline Culture & 0.73 & 0.31 & 0.00 & 96 & 1.00 & 4.67 & 2.54 & 0.81 & 0.66 & 55 & 1.00 & 3.67 & 2.04 & 0.68 & 0.46 \\
\hline Employee & 0.43 & 0.30 & 0.00 & 96 & 1.00 & 5.00 & 2.97 & 0.98 & 0.96 & 55 & 1.00 & 4.50 & 2.35 & 0.86 & 0.74 \\
\hline Skills & 0.60 & 0.26 & 0.00 & 96 & 1.50 & 5.00 & 3.49 & 0.66 & 0.44 & 55 & 1.75 & 4.50 & 3.11 & 0.65 & 0.42 \\
\hline
\end{tabular}




\section{Discussion}

The overall findings supported the assumption that non-digitalized enterprises perceive higher barriers when compared to digitalized enterprises. On a more detailed level, differences in the degrees and patterns are visible. In the following, we will therefore discuss the quantitative findings in light of scientific literature and open-ended qualitative responses in the questionnaire.

When looking at the leadership dimension, strategy work and DT awareness are organizational determinants of contextual conditions, affecting a DT early on [20]. A culture of organizational learning is essential to build up awareness and cope with a DT. One method to implement organizational learning is the employment of projects [46]. The results demonstrated a higher barrier perception for the concept of organizational learning in the non-digitalized subset. Here, a dedicated roadmap with explicit projects to learn more about DT might serve as a tool to alleviate this barrier. Both groups state age structure as a hinder to awareness in the open answers. The digitalized group reports the need for specific training. Another dimension to account for material determinants of contextual conditions is skills. With the diffusion of digital technologies, employees need to develop specific knowledge and skills. the perceptions about DT's potential demonstrated the highest delta for the means in the skills dimension. Surprisingly, however, both standard deviations for the skills dimension in Table 4 were rather similar, showing that employees in both subsets had the same perception about their respective mean perception. Minor differences between the subsets existed in the training dimension. It seemed that employees in both subsets perceived the training opportunities as limited. Nevertheless, respondents from digitalized enterprises report more specific training needs in the open answers. Both groups would benefit from training with different specificity [47]. Contextual conditions affect mechanisms [20]. Our dimension leadership represents aspects of mechanism. Leadership shows high differences between the mean figures and rather high effect size in aggerated dimensions presented in Table 4. From the additional qualitative data, employees of digitalized enterprises tend to question the honesty of DT initiatives. Several studies proposed the concept of a Digital Business Strategy as a new perspective [5], which serves as an innovative mechanism [20]. Especially in times of change, adequate leadership and communication are essential to overcome the barriers [48]. Another aspect of the non-readiness of the organizations for DT is the lack of dedicated roles which coincides with changes in the organizational setup [20]. Certain authors proposed the introduction of the role of a CDO or to evolve the role of the Chief Information Officer to include the above-mentioned duties [49]. Of course, these roles need support from the existing roles and other new roles within the organizational hierarchy. Also, in the employee dimension, the highest standard deviation was observed in the non-digitalized subset. These participants exhibited perceptions about available resources. The non-digitalized group is more worried about a mismatch of merging employees' interests and DT. The dimension DT itself is also part of the outcome within the organizational setup as it represents a product perspective [20]. As the DT dimension was our selection criteria, the means of the digitalized group is higher. In general, the digitalized group shows a higher degree of specify, which is important especially within the contextual conditions. If these are set right, they will influence further work on DT in a positive way until one desired outcome is a "smart, connected and customized product" [20]. Digitalized enterprises are working to redefine their value propositions [18] as they strive to become data-driven enterprises [21].

The quantitative analysis has shown that digitalized enterprises have been able to overcome some barriers and have presumably been able to drive their DT as a result. Nevertheless, some organizational barriers still exist today. However, less digitalized enterprises could benefit from this insight. While overcoming barriers requires effort, focusing on the key barriers as compared to simultaneously addressing all could be a more efficient path to a digitalized enterprise. Looking at the biggest differences between the two data sets, defining and communicating a clear DT strategy and specifying roles to manage DT projects seems to be a good starting point for non-digitalized enterprises.

\section{Conclusion and limitations}

In the present study, we systematically identified and quantified the impact of barriers on DT based on a research approach that utilized subsets. In the preparation stage, we collected qualitative data on obstacles and barriers from several corporations of various sizes and industries, and we then transferred these data into a measurement model [14]. The resulting questionnaire was employed to survey German engineering enterprises, as this industry has been traditionally at the focus of the German economy [12]. Thus, engineering enterprises have been the target in several government initiatives that attempted to foster DT [13]. The subsets allowed us to specifically compare and reflect on differences between DT processes and associated barriers in detail. Interestingly, the perceived barriers in the non-digitalized subset were higher with a higher degree of disagreement. Essential elements of the DT such as smart products and services as well as 
digitally enhanced business processes exhibited a higher perception in the subset of digitalized enterprises. Thus, our study contributes to a deeper understanding of the behavior in different subsets. By researching the barriers, we contribute to barrier research and research on success factors. In fact, once barriers are removed, they no longer hinder the DT process. The commonly encountered barriers across the industry could even become success factors and competitive advantages.

We used a valid method to identify the subsets [41]. There are other statistical procedures such as clustering techniques to determine the number of subsets. These clustering techniques can be explorative or the number of subsets needs to be specified before [50]. In larger datasets, the properties of our subsets might change. Future studies should focus on broadening the data set and the identification of further subsets. These subsets could be aligned to an empirically grounded maturity model, which is our approach, too. Klötzer and Pflaum set up a matrix for the determination of four fields [21], which we aggregated into two. From a sampling perspective, the assignment of the respondents via social media could be problematic, as the sample might demonstrate bias and even underestimate the barriers. Social networks might provoke a filter bubble [51] and not every employee might be active on these networks. Since our study aimed to measure perceptions about DT and associated barriers, recruitment in professional digital networks was considered a valid sampling strategy. We suppose tech-savvy employees are active on such networks. DT is a trend discussed in several professional journals. Thus, we assumed that our participants were aware of this term. We developed our items based on interviews in connection with theoretical frameworks. Further item validations are needed to determine if the items cover all aspects of the dimensions. Also, we focused on the automotive and engineering industries due to their significance in the German economy. It might be especially interesting to expand this research into more service-oriented industries. Products and services exhibit significant differences in their characteristics and value creation methods. Also, products are supposed to be enriched by services leading to a higher degree of servitization [47]. A comparison of the subsets in other industries might broaden the perspective for new insights.

\section{References}

[1] Schwab, K., The fourth industrial revolution, Crown Business, New York, 2017.

[2] Brynjolfsson, E., and A. McAfee, The second machine age: Work, progress, and prosperity in a time of brilliant technologies, W. W. Norton \& Company, New York, NY, 2014.
[3] Mithas, S., A. Tafti, and W. Mitchell, "How a firm's competitive environment and digital strategy posture influence digital business strategy", Management Information Systems Quarterly 37(2), 2013, pp. 511536.

[4] Dery, K., I.M. Sebastian, and N. van der Meulen, "The Digital Workplace is Key to Digital Innovation", MIS QUARTERLY EXECUTIVE 16(2), 2017, pp. 135-152.

[5] Bharadwaj, A., O. El Sawy, P. Pavlou, and N. Venkatraman, "Digital business strategy: toward a next generation of insights", Management Information Systems Quarterly 37(2), 2013, pp. 471-482.

[6] Nguyen, D.K., Broekhuizen, J.Q. Dong, and P.C. Verhoef, "Digital readiness: construct development and empirical validation", ICIS 2019 Proceedings, 2019.

[7] Vial, G., "Understanding digital transformation: A review and a research agenda", The Journal of Strategic Information Systems 28(2), 2019, pp. 118-144.

[8] Vogelsang, K., K. Liere-Netheler, S. Packmohr, and U. Hoppe, "Barriers to Digital Transformation in Manufacturing: Development of a Research Agenda", Proceedings of the 52nd Hawaii International Conference on System Sciences, (2019), 4937-4946.

[9] Porter, M.E., "What is Strategy?", Harvard Business Review, 1996, 61-78.

[10] Pabst von Ohain, B., "Leader Attributes for Successful Digital Transformation", ICIS 2019 Proceedings, 2019.

[11] Chi, M., X. Lu, J. Zhao, and Y. Li, "The impacts of digital business strategy on firm performance: the mediation analysis of e-collaboration capability", International Journal of Information Systems and Change Management 10(2), 2018, pp. 123.

[12] destatis.de, "Economic Sectors and Enterprises: Industry, Manufacturing", 2020. https://www.destatis.de/EN/Themes/Economic-SectorsEnterprises/Industry-Manufacturing/_node.html

[13] Kagermann, H., W. Wahlster, and J. Helbig, Recommendations for implementing the strategic initiative Industrie 4.0, acatech - National Academy of Science and Engineering, 2013.

[14] MacKenzie, S.B., P.M. Podsakoff, and N.P. Podsakoff, "Construct Measurement and Validation Procedures in MIS and Behavioral Research: Integrating New and Existing Techniques", MIS Quarterly 35(2), 2011, pp. 293-334.

[15] Hirsch-Kreinsen, H., "Digitization of industrial work: development paths and prospects", Journal for Labour Market Research 49(1), 2016, pp. 1-14.

[16] Leonardi, P.M., "Materiality, Sociomateriality, and Socio-Technical Systems: What Do These Terms Mean? How are They Related? Do We Need Them?", In Materiality and Organizing: Social Interaction in a Technological World. OUP Oxford, 2012, 384.

[17] Reis, J., M. Amorim, N. Melão, and P. Matos, "Digital Transformation: A Literature Review and Guidelines for Future Research", Trends and Advances in Information Systems and Technologies, Springer International Publishing (2018), 411-421.

[18] Wessel, L., A. Baiyere, R. Ologeanu-Taddei, et al., "Unpacking the Difference Between Digital Transformation and IT-Enabled Organizational 
Transformation", Journal of the Association for Information Systems 22(1), 2021, pp. 102-129.

[19] Vukšić, V.B., L. Ivančić, and D.S. Vugec, “A Preliminary Literature Review Of Digital Transformation Case Studies", 2018.

[20] Hanelt, A., R. Bohnsack, D. Marz, and C. Antunes Marante, "A Systematic Review of the Literature on Digital Transformation: Insights and Implications for Strategy and Organizational Change", Journal of Management Studies 58(5), 2021, pp. 1159-1197.

[21] Klötzer, C., and A. Pflaum, "Toward the development of a maturity model for digitalization within the manufacturing industry's supply chain", Proceedings of the 50th Hawaii International Conference on System Sciences, (2017), 4210-4219.

[22] Piatier, A., Barriers to innovation, F. Pinter, London; Dover, N.H, 1984.

[23] Bilgeri, D., and F. Wortmann, "Barriers to IoT Business Model Innovation", Proceedings of the 13th International Conference on Wirtschaftsinformatik, 2017, pp. 987990.

[24] Demirbas, D., J.G. Hussain, and H. Matlay, "Ownermanagers' perceptions of barriers to innovation: empirical evidence from Turkish SMEs", Journal of Small Business and Enterprise Development 18(4), 2011, pp. 764-780.

[25] Madrid-Guijarro, A., D. Garcia, and H. Van Auken, "Barriers to Innovation among Spanish Manufacturing SMEs", Journal of Small Business Management 47(4), 2009, pp. 465-488.

[26] Jones, M.D., S. Hutcheson, and J.D. Camba, "Past, present, and future barriers to digital transformation in manufacturing: A review", Journal of Manufacturing Systems 60, 2021, pp. 936-948.

[27] Henriette, E., M. Feki, I. Boughzala, and others, "Digital Transformation Challenges.", Mediterranean Conference on Information Systems (MICS), (2016), 1-7.

[28] Chesbrough, H., "Business Model Innovation: Opportunities and Barriers", Long Range Planning 43(2), 2010, pp. 354-363.

[29] Dremel, C., "Barriers to the adoption of big data analytics in the automotive sector", Proceedings of the 23rd American Conference on Information Systems (AMCIS), (2017), 1-10.

[30] Aguiar, T., S. Bogea Gomes, P. Rupino da Cunha, and M. Mira da Silva, "Digital Transformation Capability Maturity Model Framework", 2019 IEEE 23rd International Enterprise Distributed Object Computing Conference (EDOC), IEEE (2019), 51-57.

[31] Helms, M.M., and J. Nixon, "Exploring SWOT analysis - where are we now? A review of academic research from the last decade", Journal of Strategy and Management 3(3), 2010, pp. 215-251.

[32] Hadjimanolis, A., "The Barriers Approach to Innovation", In L.V. Shavinina, ed., The International Handbook on Innovation. Pergamon, Oxford, 2003, 559573.

[33] Akram, M.S., "Drivers and barriers to online shopping in a newly digitalized society", TEM Journal 7(1), 2018, pp. 118-127.
[34] Creswell, J.W., A concise introduction to mixed methods research, SAGE, Los Angeles, 2015.

[35] Mayring, P., Qualitative content analysis: theoretical foundation, basic procedures and software solution, Beltz Verlag, Klagenfurt, 2014.

[36] Bryman, A., Social Research Methods, Oxford University Press, 2016.

[37] Bouckenooghe, D., G. Devos, and H. Van den Broeck, "Organizational Change Questionnaire-Climate of Change, Processes, and Readiness: Development of a New Instrument", The Journal of Psychology 143(6), 2009, pp. 559-599.

[38] Yin, R.K., Case study research: design and methods, SAGE, Los Angeles, 2014.

[39] Taber, K.S., "The Use of Cronbach's Alpha When Developing and Reporting Research Instruments in Science Education", Research in Science Education 48(6), 2018, pp. 1273-1296.

[40] Pallant, J., SPSS survival manual: a step by step guide to data analysis using SPSS for Windows (version 12), Open University Press, Maidenhead, Berkshire. U.K.; New York, NY, 2005.

[41] Kaňovská, L., "Are smart service manufacturing providers different in cooperation and innovation flexibility, in innovation performance and business performance from non-smart service manufacturing providers?", Engineering Management in Production and Services 12(4), 2020, pp. 105-116.

[42] Štemberger, M.I., J. Erjavec, A. Manfreda, and J. Jaklič, "PATTERNS OF APPROACHES TO DIGITAL TRANSFORMATION: AN INSTITUTIONAL ARRANGEMENTS PERSPECTIVE", Economic and Business Review 21(3), 2019, pp. 467-492.

[43] Coolican, H., Research methods and statistics in psychology, Psychology Press, Taylor \& Francis Group, London; New York, 2014.

[44] Fritz, C.O., P.E. Morris, and J.J. Richler, "Effect size estimates: Current use, calculations, and interpretation.", Journal of Experimental Psychology: General 141(1), 2012, pp. 2-18.

[45] Cohen, J., "Statistical Power Analysis", Current Directions in Psychological Science 1(3), 1992, pp. 98101.

[46] Gareis, R., "Changes of organizations by projects", International Journal of Project Management 28(4), 2010, pp. 314-327.

[47] Marcon, É., A. Marcon, M.-A. Le Dain, N.F. Ayala, A.G. Frank, and J. Matthieu, "Barriers for the digitalization of servitization", Procedia CIRP, (2019), 254-259.

[48] Lauer, T., Change Management: Fundamentals and Success Factors, Springer Berlin Heidelberg, Berlin, Heidelberg, 2021.

[49] Earley, S., "The Evolving Role of the CDO", IT Professional 19(1), 2017, pp. 64-69.

[50] Jain, A.K., "Data clustering: 50 years beyond K-means", Pattern Recognition Letters 31(8), 2010, pp. 651-666.

[51] Matakos, A., and A. Gionis, "Tell me Something My Friends do not Know: Diversity Maximization in Social Networks", 2018 IEEE International Conference on Data Mining (ICDM), IEEE (2018), 327-336. 